\title{
Co-hydrotreatment of Bio-oil Lignin-rich Fraction and Vegetable Oil
}

\author{
Yinglei Han ${ }^{1}$, Anamaria P.P. Pires ${ }^{1}$, Manuel Garcia-Perez ${ }^{1,2 *}$ \\ ${ }^{1}$ Biological Systems Engineering Department, Washington State University, Pullman, WA, \\ 99163 \\ ${ }^{2}$ Bio-products Sciences and Engineering Laboratory, Washington State University-Tri-cities, \\ Richland, 99354
}




\section{Detail information by GC-MS on the cohydrotreated oils}

GC/MS of the analysis on the volatile compounds in the hydrotreated oils were carried out as shown in the Figure S1. The spectrums were normalized to make an explicit comparison on the product profiles in between these hydrotreated oils with or without the presence of 1-butanol during cohydrotreatment.
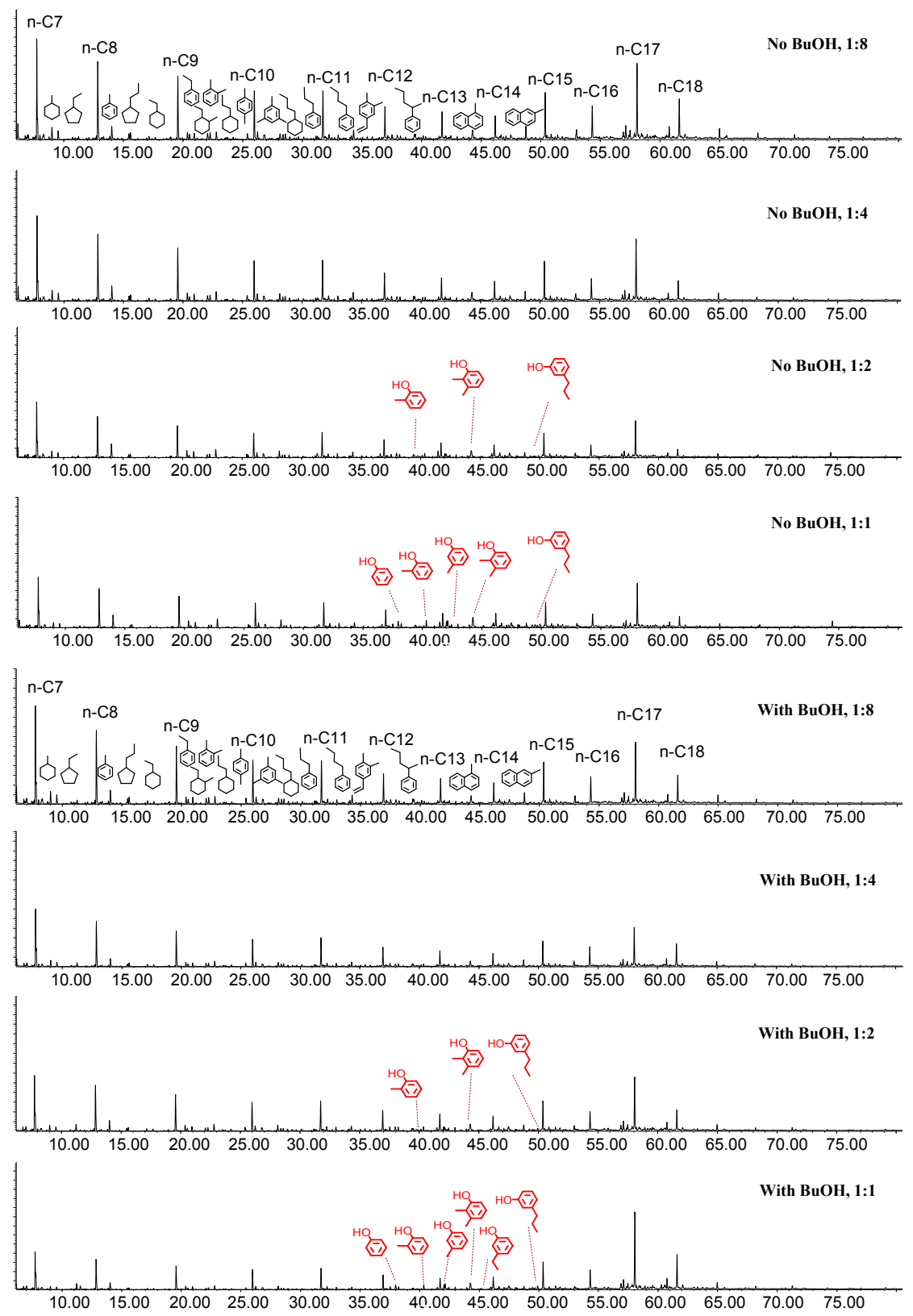

Figure S1. GCMS on all the hydrotreated oils from the coprocessing 


\section{Identification of the major compounds of the oil from hydrotreating canola oil alone}

(same condition as other cohydrotreatment)

Figure S2 shows the GCMS spectrum of the hydrotreated oil from canola oil alone (no LRO or 1butanol). The major compounds had been identified here (Table S1) are very similar to the ones from cohydrotreatment abovementioned. It is worth to note that, even without LRO, many aromatics were detected from this hydrotreated oil. This implies that a self-aromatization possibly occurred over the $\mathrm{CoMo} / \mathrm{A} 12 \mathrm{O} 3$ catalyst.

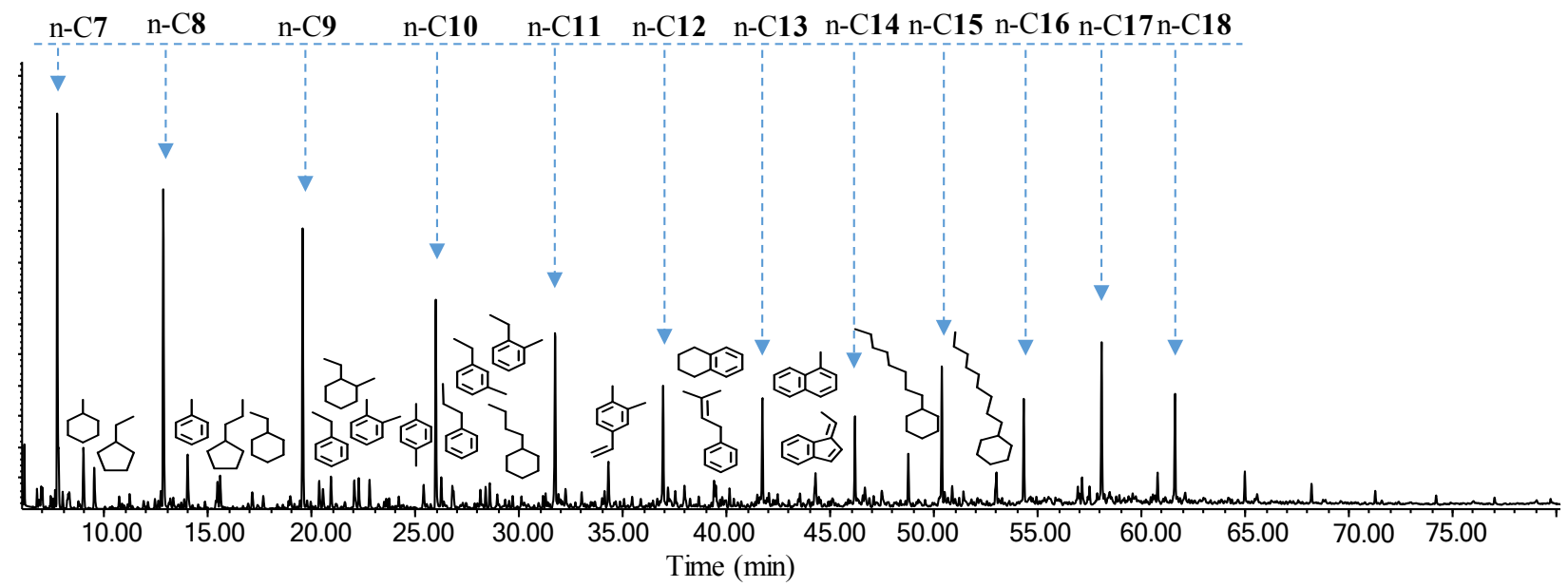

Figure S2. GCMS on all the hydrotreated oils from canola oil only (same condition with the coprocessing) 
Table S1. The identified compound from hydrotreated canola oil (same condition with the coprocessing)

\begin{tabular}{|c|c|c|c|}
\hline Peak ID & $\mathrm{RT}$ (min) & Name & Formula \\
\hline 1 & 4.609 & Pentane & $\mathrm{C}_{5} \mathrm{H}_{12}$ \\
\hline 2 & 6.162 & Cyclopentane, methyl- & $\mathrm{C}_{6} \mathrm{H}_{12}$ \\
\hline 3 & 7.003 & Hexane, 3-methyl- & $\mathrm{C}_{7} \mathrm{H}_{16}$ \\
\hline 4 & 7.74 & Heptane & $\mathrm{C}_{7} \mathrm{H}_{16}$ \\
\hline 5 & 7.803 & Cyclohexene & $\mathrm{C}_{6} \mathrm{H}_{10}$ \\
\hline 6 & 9.015 & Cyclohexane, methyl- & $\mathrm{C}_{7} \mathrm{H}_{14}$ \\
\hline 7 & 9.553 & Cyclopentane, ethyl- & $\mathrm{C}_{7} \mathrm{H}_{14}$ \\
\hline 8 & 11.237 & Heptane, 3-methyl- & $\mathrm{C}_{8} \mathrm{H}_{18}$ \\
\hline 9 & 12.891 & Octane & $\mathrm{C}_{8} \mathrm{H}_{18}$ \\
\hline 10 & 14.027 & Toluene & $\mathrm{C}_{7} \mathrm{H}_{8}$ \\
\hline 11 & 15.479 & Cyclopentane, propyl- & $\mathrm{C}_{8} \mathrm{H}_{16}$ \\
\hline 12 & 15.592 & Cyclohexane, ethyl- & $\mathrm{C}_{8} \mathrm{H}_{16}$ \\
\hline 13 & 17.158 & Octane, 4-methyl- & $\mathrm{C}_{9} \mathrm{H}_{20}$ \\
\hline 14 & 19.581 & Nonane & $\mathrm{C}_{9} \mathrm{H}_{20}$ \\
\hline 15 & 20.364 & Ethylbenzene & $\mathrm{C}_{8} \mathrm{H}_{10}$ \\
\hline 16 & 20.566 & Cyclohexane, 1-ethyl-2-methyl-, cis- & $\mathrm{C}_{9} \mathrm{H}_{18}$ \\
\hline 17 & 20.97 & o-Xylene & $\mathrm{C}_{8} \mathrm{H}_{10}$ \\
\hline 18 & 22.068 & Cyclohexane, propyl- & $\mathrm{C}_{9} \mathrm{H}_{18}$ \\
\hline 19 & 22.296 & Cyclopentane, butyl- & $\mathrm{C}_{9} \mathrm{H}_{18}$ \\
\hline 20 & 22.801 & p-Xylene & $\mathrm{C}_{8} \mathrm{H}_{10}$ \\
\hline 21 & 23.243 & Octane, 4-ethyl- & $\mathrm{C}_{10} \mathrm{H}_{22}$ \\
\hline 22 & 23.747 & Nonane, 2-methyl- & $\mathrm{C}_{10} \mathrm{H}_{22}$ \\
\hline 23 & 24.215 & Octane, 2,6-dimethyl- & $\mathrm{C}_{10} \mathrm{H}_{22}$ \\
\hline 24 & 25.401 & Cyclodecane & $\mathrm{C}_{10} \mathrm{H}_{20}$ \\
\hline 25 & 25.982 & Decane & $\mathrm{C}_{10} \mathrm{H}_{22}$ \\
\hline 26 & 26.247 & Benzene, propyl- & $\mathrm{C}_{9} \mathrm{H}_{12}$ \\
\hline 27 & 26.84 & Benzene, 1-ethyl-3-methyl- & $\mathrm{C}_{9} \mathrm{H}_{12}$ \\
\hline 28 & 27.863 & Cyclohexane, 1-methyl-2-propyl- & $\mathrm{C}_{10} \mathrm{H}_{20}$ \\
\hline 29 & 28.141 & Benzene, 1-ethyl-2-methyl- & $\mathrm{C}_{9} \mathrm{H}_{12}$ \\
\hline 30 & 28.381 & Cyclohexane, butyl- & $\mathrm{C}_{10} \mathrm{H}_{20}$ \\
\hline 31 & 28.583 & Cyclopentane, pentyl- & $\mathrm{C}_{10} \mathrm{H}_{20}$ \\
\hline 32 & 28.949 & Benzene, 1,2,3-trimethyl- & $\mathrm{C}_{9} \mathrm{H}_{12}$ \\
\hline 33 & 29.327 & Decane, 5-methyl- & $\mathrm{C}_{11} \mathrm{H}_{24}$ \\
\hline 34 & 29.517 & Decane, 4-methyl- & $\mathrm{C}_{11} \mathrm{H}_{24}$ \\
\hline 35 & 29.694 & Decane, 2-methyl- & $\mathrm{C}_{11} \mathrm{H}_{24}$ \\
\hline 36 & 30.123 & Decane, 3-methyl- & $\mathrm{C}_{11} \mathrm{H}_{24}$ \\
\hline 37 & 31.158 & Cyclotridecane & $\mathrm{C}_{13} \mathrm{H}_{26}$ \\
\hline 38 & 31.272 & 5-Undecene & $\mathrm{C}_{11} \mathrm{H}_{22}$ \\
\hline 39 & 31.739 & Undecane & $\mathrm{C}_{11} \mathrm{H}_{24}$ \\
\hline 40 & 31.903 & Benzene, 1-methyl-3-propyl- & $\mathrm{C}_{10} \mathrm{H}_{14}$ \\
\hline 41 & 32.231 & Benzene, butyl- & $\mathrm{C}_{10} \mathrm{H}_{14}$ \\
\hline 42 & 32.471 & Cyclopropane, 1-pentyl-2-propyl- & $\mathrm{C}_{11} \mathrm{H}_{22}$ \\
\hline 43 & 33.014 & Benzene, 1-methyl-2-propyl- & $\mathrm{C}_{10} \mathrm{H}_{14}$ \\
\hline 44 & 33.658 & Benzene, 1-methyl-3-(1-methylethyl)- & $\mathrm{C}_{10} \mathrm{H}_{14}$ \\
\hline 45 & 34.001 & Benzene, 1-methyl-4-(1-methylethyl)- & $\mathrm{C}_{10} \mathrm{H}_{14}$ \\
\hline 46 & 34.112 & Cyclohexane, pentyl- & $\mathrm{C}_{11} \mathrm{H}_{22}$ \\
\hline 47 & 34.289 & Benzene, 4-ethenyl-1,2-dimethyl- & $\mathrm{C}_{10} \mathrm{H}_{12}$ \\
\hline
\end{tabular}


Table S1. The identified compound from hydrotreated canola oil (same condition with the coprocessing) Continuation......

\begin{tabular}{|c|c|c|c|}
\hline Peak ID & $\mathrm{RT}$ (min) & Name & Formula \\
\hline 48 & 34.655 & 2,6-Dimethyldecane & $\mathrm{C}_{12} \mathrm{H}_{26}$ \\
\hline 49 & 34.844 & 2,3-Dimethyldecane & $\mathrm{C}_{12} \mathrm{H}_{26}$ \\
\hline 50 & 35.046 & Undecane, 2-methyl- & $\mathrm{C}_{12} \mathrm{H}_{26}$ \\
\hline 51 & 35.438 & Undecane, 3-methyl- & $\mathrm{C}_{12} \mathrm{H}_{26}$ \\
\hline 52 & 36.927 & Dodecane & $\mathrm{C}_{12} \mathrm{H}_{26}$ \\
\hline 53 & 37.521 & Benzene, pentyl- & $\mathrm{C}_{11} \mathrm{H}_{16}$ \\
\hline 54 & 37.975 & Indan, 4-methyl- & $\mathrm{C}_{10} \mathrm{H}_{12}$ \\
\hline 55 & 38.228 & Benzene, (1-methylbutyl)- & $\mathrm{C}_{11} \mathrm{H}_{16}$ \\
\hline 56 & 38.657 & Tetralin & $\mathrm{C}_{10} \mathrm{H}_{12}$ \\
\hline 57 & 39.389 & Benzene, (3-methyl-2-butenyl)- & $\mathrm{C}_{11} \mathrm{H}_{14}$ \\
\hline 58 & 39.478 & Cyclododecane & $\mathrm{C}_{12} \mathrm{H}_{24}$ \\
\hline 59 & 39.78 & Indan, 1-ethyl- & $\mathrm{C}_{11} \mathrm{H}_{14}$ \\
\hline 60 & 40.134 & 1,6-Dimethylindan & $\mathrm{C}_{11} \mathrm{H}_{14}$ \\
\hline 61 & 41.472 & Naphthalene & $\mathrm{C}_{10} \mathrm{H}_{8}$ \\
\hline 62 & 41.725 & Tridecane & $\mathrm{C}_{13} \mathrm{H}_{28}$ \\
\hline 63 & 42.444 & Benzene, hexyl- & $\mathrm{C}_{12} \mathrm{H}_{18}$ \\
\hline 64 & 43 & Benzene, (1,3-dimethylbutyl)- & $\mathrm{C}_{12} \mathrm{H}_{18}$ \\
\hline 65 & 43.53 & 6-Methyltetralin & $\mathrm{C}_{11} \mathrm{H}_{14}$ \\
\hline 66 & 44.275 & Cyclotridecane & $\mathrm{C}_{13} \mathrm{H}_{26}$ \\
\hline 67 & 45.866 & Naphthalene, 5-ethyl-1,2,3,4-tetrahydro- & $\mathrm{C}_{12} \mathrm{H}_{16}$ \\
\hline 68 & 46.181 & Tetradecane & $\mathrm{C}_{14} \mathrm{H}_{30}$ \\
\hline 69 & 46.661 & Naphthalene, 1-methyl- & $\mathrm{C}_{11} \mathrm{H}_{10}$ \\
\hline 70 & 47.077 & Benzene, heptyl- & $\mathrm{C}_{13} \mathrm{H}_{20}$ \\
\hline 71 & 47.481 & 1H-Indene, 1-ethylidene- & $\mathrm{C}_{11} \mathrm{H}_{10}$ \\
\hline 72 & 48.756 & Cyclotetradecane & $\mathrm{C}_{14} \mathrm{H}_{28}$ \\
\hline 73 & 50.347 & Pentadecane & $\mathrm{C}_{15} \mathrm{H}_{32}$ \\
\hline 74 & 50.865 & Naphthalene, 1-ethyl- & $\mathrm{C}_{12} \mathrm{H}_{12}$ \\
\hline 75 & 51.408 & Benzene, octyl- & $\mathrm{C}_{14} \mathrm{H}_{22}$ \\
\hline 76 & 52.986 & n-Nonylcyclohexane & $\mathrm{C}_{15} \mathrm{H}_{30}$ \\
\hline 77 & 54.311 & Hexadecane & $\mathrm{C}_{16} \mathrm{H}_{34}$ \\
\hline 78 & 57.114 & 3-Heptadecene, (Z)- & $\mathrm{C}_{17} \mathrm{H}_{34}$ \\
\hline 79 & 58.061 & Heptadecane & $\mathrm{C}_{17} \mathrm{H}_{36}$ \\
\hline 80 & 60.762 & Cyclodecane, octyl- & $\mathrm{C}_{18} \mathrm{H}_{36}$ \\
\hline 81 & 61.595 & Octadecane & $\mathrm{C}_{18} \mathrm{H}_{38}$ \\
\hline 82 & 64.979 & Nonadecane & $\mathrm{C}_{19} \mathrm{H}_{40}$ \\
\hline 83 & 68.198 & Eicosane & $\mathrm{C}_{20} \mathrm{H}_{42}$ \\
\hline 84 & 71.266 & Heneicosane & $\mathrm{C}_{21} \mathrm{H}_{44}$ \\
\hline 85 & 71.607 & 1H-Cyclopropa[1]phenanthrene,1a,9b-dihydro- & $\mathrm{C}_{15} \mathrm{H}_{12}$ \\
\hline 86 & 79.068 & Pyrene & $\mathrm{C}_{16} \mathrm{H}_{10}$ \\
\hline
\end{tabular}




\section{Validation on the UV-fluorescence analysis}

To validate the analysis of the UV-fluorescence, the possibility of self-adsorption on each sample needs to be excluded. According to Figure S3, similar profile can be observed in the fluorescence spectrums at different concentrations from $10 \mathrm{ppm}$ to $200 \mathrm{ppm}$. In this study, $20 \mathrm{ppm}$ was selected for all the samples tested by the UV-fluorescence. In a very dilute situation, such as $10 \mathrm{ppm}$, the oligomer peaks still can be observed (at around $370-550 \mathrm{~nm}$ ). This can validate that the similar peaks presented at concentration of $20 \mathrm{ppm}$ should be linked to the fluorescence emission of the oligomers, not self-adsorption.

In addition, the major peaks of interest were labelled as A, B, C, D, E, and F as shown in Figure S3. A linear trend with good fitting was observed for all these major peaks from 0 to $20 \mathrm{ppm}$.

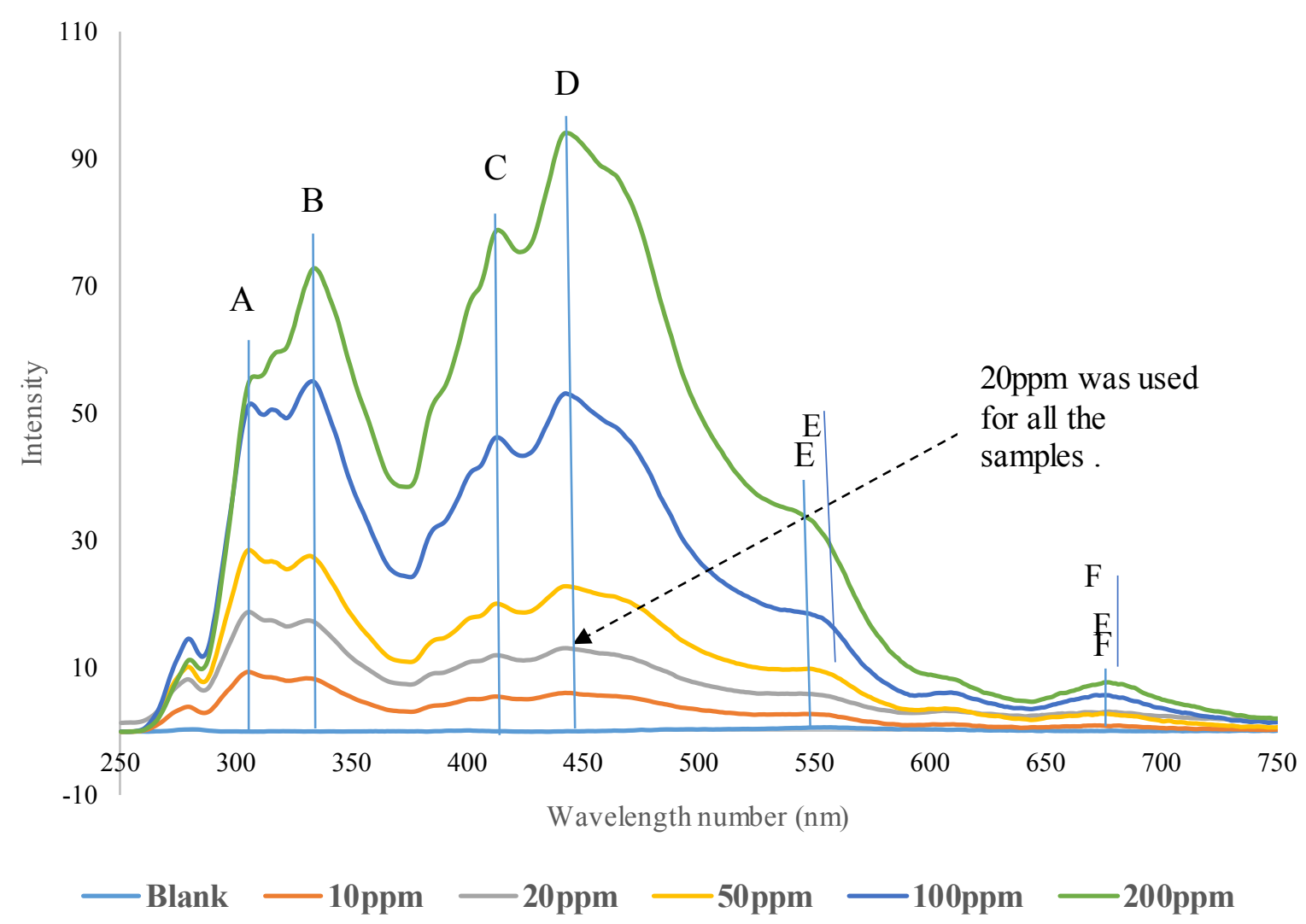

Figure S3. UV-Fluorescence on one sample at different concentration (10-200pmm). The sample here was the hydrotreated oil at the canola/LRO ratio 1:1 in the presence of 1-butanol. 


\section{Additional characterization on the hydrotreated oils: carbonyl content}

Figure S4 shows a comparison on carbonyl content of the initial blends and their corresponding hydrotreated oils is performed. It's worth to note that the resulting carbonyls was not only derived from the LRO side but also from incomplete decarbonylation of the triglycerides in canola oil. Overall, a reduction of the carbonyl content after hydrotreatment was observed. The carbonyl group, extensively presented in bio-oil, has been identified as an active group for the coke formation during bio-oil hydrotreatement ${ }^{1,2}$. Even though many carbonyl-containing compounds had been removed in the extraction, the resulting LRO should still contain some carbonyl on the alkylated branched of phenols. Therefore, it's important to track the change of carbonyl content of the LRO after cohydrotreatment.

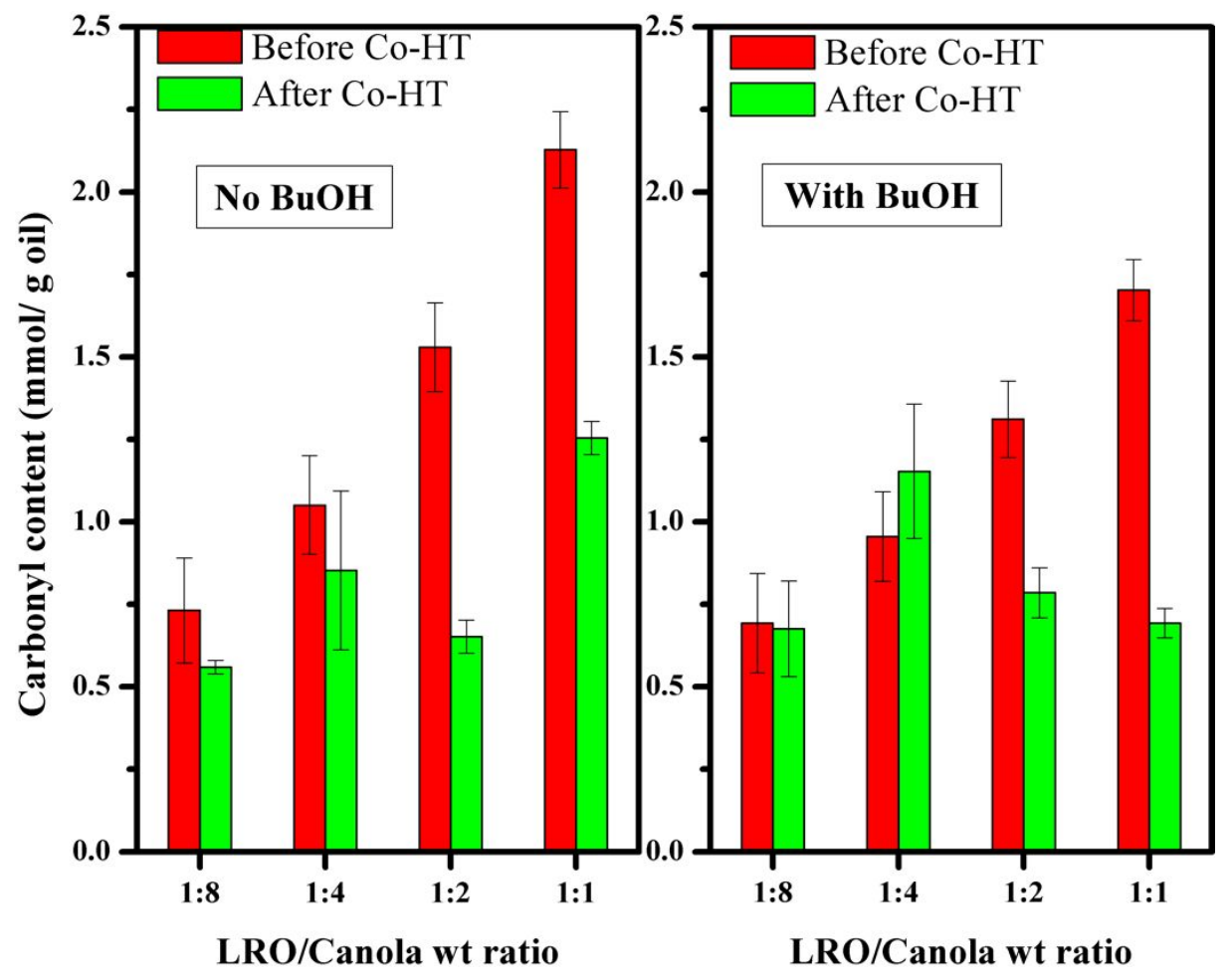

Figure S4. Carbonyl content of the LRO/Canola blends and the corresponding hydrotreated oils 


\section{References}

1. Wang, H.; Lee, S.-J.; Olarte, M. V.; Zacher, A. H., Bio-oil Stabilization by Hydrogenation over Reduced Metal Catalysts at Low Temperatures. ACS Sustainable Chemistry \& Engineering 2016, 4, (10), 5533-5545.

2. Han Y, Stankovikj F, Garcia-Perez M: Co-hydrotreatment of tire pyrolysis oil and vegetable oil for the production of transportation fuels. Fuel processing Technology, 159, 2017, 32833932. 